University of Wollongong

Research Online

Faculty of Informatics - Papers (Archive)

Faculty of Engineering and Information

Sciences

2008

\title{
Wireless interactive system for patient healthcare monitoring using mobile computing devices
}

Montserrat Ros

University of Wollongong, montse@uow.edu.au

Matthew D'Souza

CSIRO ICT Centre, Brisbane

Adam Postula

University of Queensland

Follow this and additional works at: https://ro.uow.edu.au/infopapers

Part of the Physical Sciences and Mathematics Commons

\section{Recommended Citation}

Ros, Montserrat; D'Souza, Matthew; and Postula, Adam: Wireless interactive system for patient healthcare monitoring using mobile computing devices 2008.

https://ro.uow.edu.au/infopapers/3178

Research Online is the open access institutional repository for the University of Wollongong. For further information contact the UOW Library: research-pubs@uow.edu.au 


\title{
Wireless interactive system for patient healthcare monitoring using mobile computing devices
}

\author{
Abstract \\ Recently there has been a need to incorporate the use of mobile computing devices in hospital or clinical \\ applications, to enhance patient care. The advancement of wireless technology has created unique \\ mechanisms of interaction that can meet the needs of e-health system robustness, reliability and \\ accuracy requirements. This paper presents an interactive wireless medical information system that \\ allows patient instrumentation output data to be accessed and patient details to be entered by medical \\ practitioners using mobile computing devices. We examine mobile and wireless information technology \\ concepts that can be used to interact with a medical information system for controlling real-time data \\ streaming medical instruments.

\section{Disciplines} \\ Physical Sciences and Mathematics

\section{Publication Details} \\ Ros, M., D"Souza, M. \& Postula, A. (2008). Wireless interactive system for patient healthcare monitoring \\ using mobile computing devices. IEEE International Conference on Signal Processing and \\ Communication (pp. 1-6). IEEE Explore Online: ieeexplore.ieee.org: IEEE.
}




\title{
Wireless Interactive System for Patient Healthcare Monitoring using Mobile Computing Devices
}

\author{
Montserrat Ros ${ }^{*}$, Matthew D’Souza ${ }^{\mp}$ and Adam Postula ${ }^{\dagger}$ \\ *School of Electrical, Computer and Telecommunications Engineering \\ University of Wollongong, Wollongong, Australia \\ montse_ros@uow.edu.au \\ ${ }^{\mp}$ Autonomous Systems Laboratory \\ CSIRO ICT Centre, Brisbane, Australia \\ matt.d'souza@csiro.au \\ ${ }^{\dagger}$ School of Information Technology and Electrical Engineering \\ The University of Queensland, Brisbane, Australia \\ adam@itee.uq.edu.au
}

\begin{abstract}
Recently there has been a need to incorporate the use of mobile computing devices in hospital or clinical applications, to enhance patient care. The advancement of wireless technology has created unique mechanisms of interaction that can meet the needs of e-health system robustness, reliability and accuracy requirements. This paper presents an interactive wireless medical information system that allows patient instrumentation output data to be accessed and patient details to be entered by medical practitioners using mobile computing devices. We examine mobile and wireless information technology concepts that can be used to interact with a medical information system for controlling real-time data streaming medical instruments.
\end{abstract}

Index Terms- Wireless, Telemedicine, Mobile Computing Devices

\section{INTRODUCTION}

The rapid development of wireless networks and mobile technology has lead to advances in e-health and telemedicine applications. Smart-phones and Personal Digital Assistants (PDAs) are fast becoming powerful platforms for users to interact with a variety of systems for e-health and e-work situations. Advanced mobile computing device features such as roaming web browsing or wireless network access has made it attractive to use Mobile Computing Devices for hospital and ambulatory environments. We define Mobile Computing Devices (MCDs) as standalone physical devices which use wireless technology to interface to other systems. The use of MCDs and other wireless networking technologies in e-health environments for patient record, resource or time management has the potential to improve overall patient care by reducing the occurrence of mistreatment incidents caused by faulty information. As highlighted by Bell [1] a study of medical records found that many healthcare organisations still record and distributed instrument output data and patient records in paper form, which can lead to errors in interpreting records and ultimately to misdiagnosis. Hence there is an effort to develop electronic patient record standards by a variety of e-health groups. An example is the Standard Communications Protocol (SCP) [2] and the ISO/ IEEE 11073-30300 [3] used to record instrument readings and patient details.

Wireless networks are generally used in hospitals for administration roles but are not for the monitoring of patients. Medical instruments that measure vital human measurements are generally not connected to a Local Area Network (LAN) and cannot be accessed remotely. However, having networked instruments would allow instrumentation data outputs to be recorded electronically and accessed via a LAN connection. This would allow the instrumentation output to be inserted into a patient's record which could streamline clerical data entry procedures.

We have developed a Medical Information System Network (MISN) that offers location based services such as instrument monitoring or recording and patient record entry using mobile phones. The MISN allows a medical instrument's output to be viewed and controlled by authorized mobile computing devices. Our paper examines various mobile, wireless and sensor information technology concepts that can be used to interact with a medical information system for controlling real-time data-streaming medical instruments. The MISN consists of Medical Information Nodes (MINs) connected to a LAN. The MIN is an embedded system platform that uses a Bluetooth link to communicate with mobile computing devices (MCD). Bluetooth communications was used as a large number of available MCD's are Bluetooth enabled. The short $10 \mathrm{~m}$ range of Bluetooth [4] also ensures that the MIN can only 


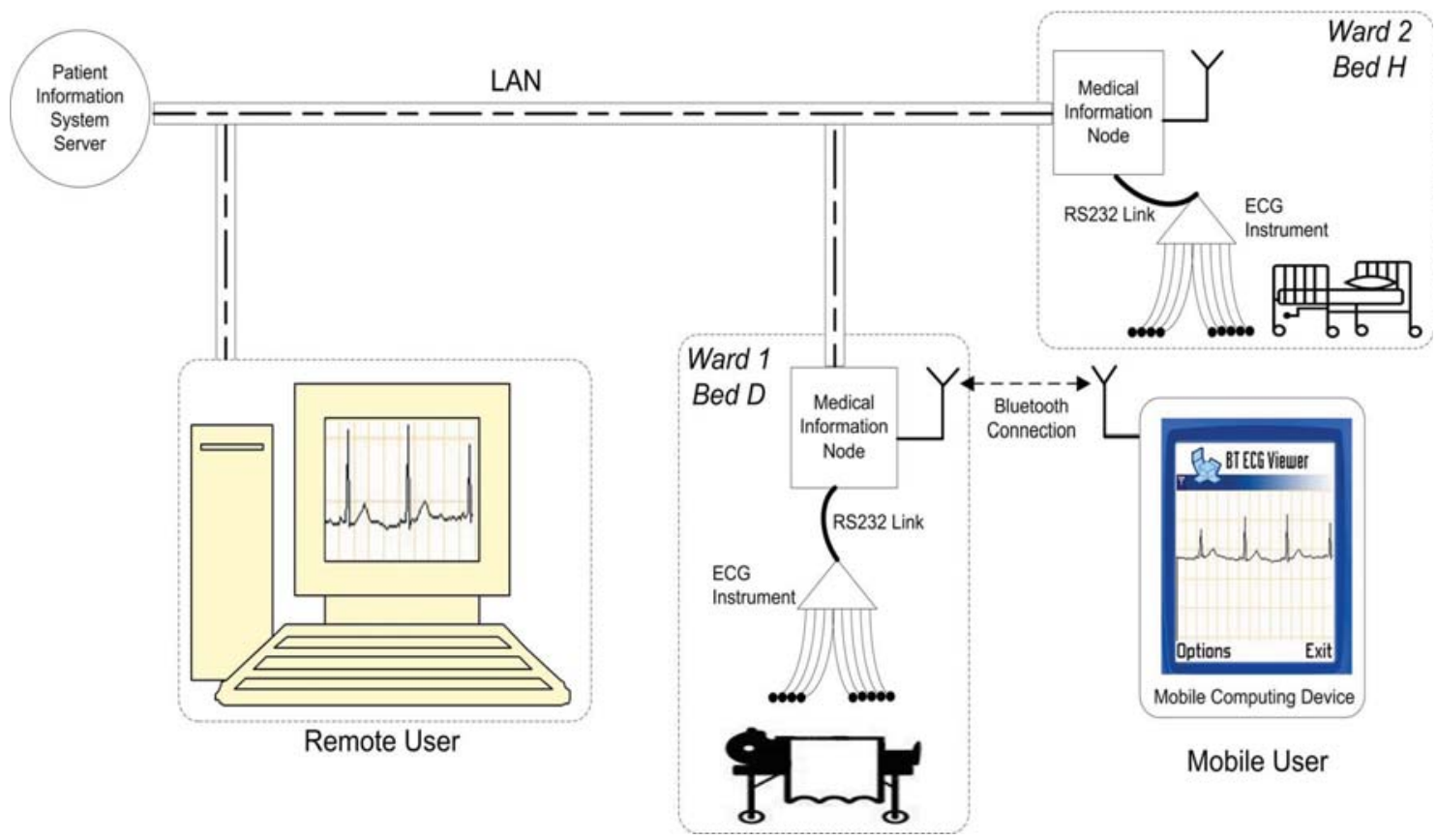

Fig. 1. Structure of Medical Information System Network (MISN)

be accessed by a user within close proximity. The MIN can also be accessed and controlled via a web interface.

This paper is organized into seven sections. Section 2 presents a review of background and related work. Section 3 presents a typical usage scenario of the medical information system. Section 4 discusses the MISN implementation. Section 5 discusses the interactive components of the medical information system. Section 6 discusses the hardware and software module implementations of the MIN. Future areas of investigation and conclusions are drawn in Section 7.

\section{BACKGROUND AND RELATED WORK}

Recent literature has focused on the use of cellular networks such as GSM as a mechanism to allow medical practitioners to access instrument and patient data. Rasid et al [5] describe a system in which Bluetooth enabled mobile phones are used to access and control an electrocardiogram (ECG) instrument. The mobile phone is connected to a patient database via a GSM network connection. The recorded output of the ECG instrument is relayed to the patient database via the mobile phone's GSM connection. One of the disadvantages of this mechanism is the transfer of the instruments output over a low data rate and potentially costly GSM connection compared to normal LAN connection.

Hung et al [6] show an implementation of a Wireless Application Protocol (WAP) based system for accessing a patient's ECG and blood pressure measurements. The patient's signals are recorded and sent via custom wireless transmitter to a receiver that stores it in a patient record database. When a user initiates a request to view a patient's record, the WAP content server accesses the patient record database.

An ECG instrument measures the human heartbeat rhythm which can help diagnose a patient's medical condition. Typically an ECG instruments requires eight electrodes and one ground electrode to be attached to a patient. Commonly used ECG instruments tend to be standalone and record the patient's ECG readings on to paper. The ECG instrument measures the difference in voltage potential between each of the electrodes. The typical ECG signal frequency range is between DC and $100 \mathrm{~Hz}$ [7]. Typical sampling speeds can be up to $500 \mathrm{~Hz}$.

\section{USER SCENARIO}

A typical user scenario involves a nurse connecting a medical monitoring instrument to a patient. For this scenario an ECG instrument is used and it requires at least two electrodes to be placed on the patient. The ECG instrument is connected to the Medical Information Node (MIN) as shown in Fig. 1. Once the nurse has ensured that the ECG instrument is properly attached to the patient, he/she then use their Mobile Computing Device (MCD) to access the MIN. The nurse can then enter various patient details such as name, ward number, etc into the various data entry fields as presented on their MCD. The output of the ECG instrument can then be viewed and checked. If the electrodes have not been placed correctly then the waveform will appear to be distorted. Usually two ECG lead waveforms (Leads I and II) are used to determine correct electrode placement. A three second display period of an ECG lead waveform is required to ensure correct electrode placement.

Once the nurse is satisfied that the ECG instrument output is within nominal accuracy, they can then select the record command on their MCD which will instruct the MIN to record the entire ECG instrument output. When the nurse selects the stop record command, the MIN will then save the recorded ECG instrument waveform output as a patient record in a folder located on the Patient Information server. The patient record is a Scalable Vector Graphics (SVG) file which will contains the patient's details as 


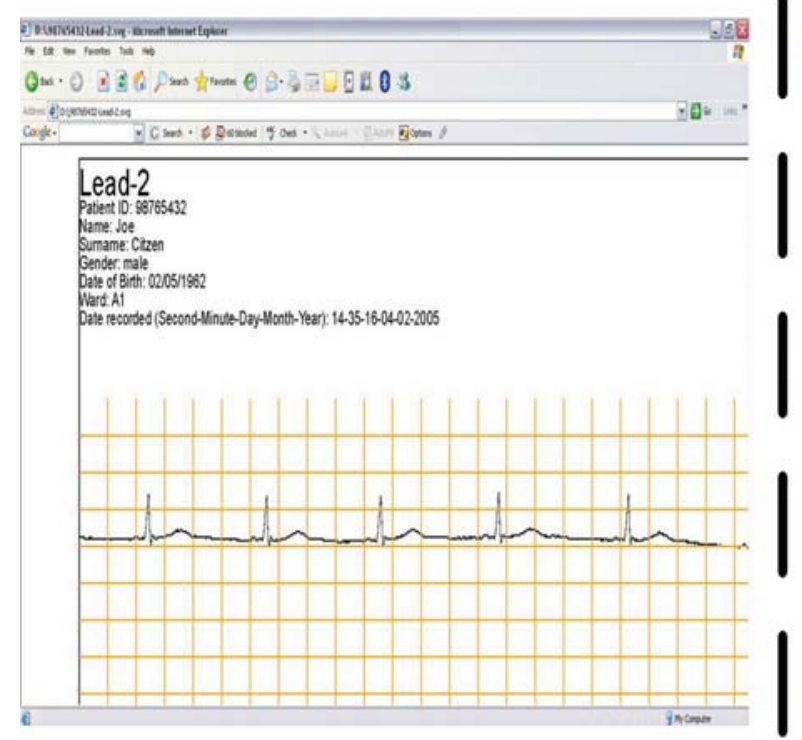

A) Patient ECG Lead Waveform SVG Record

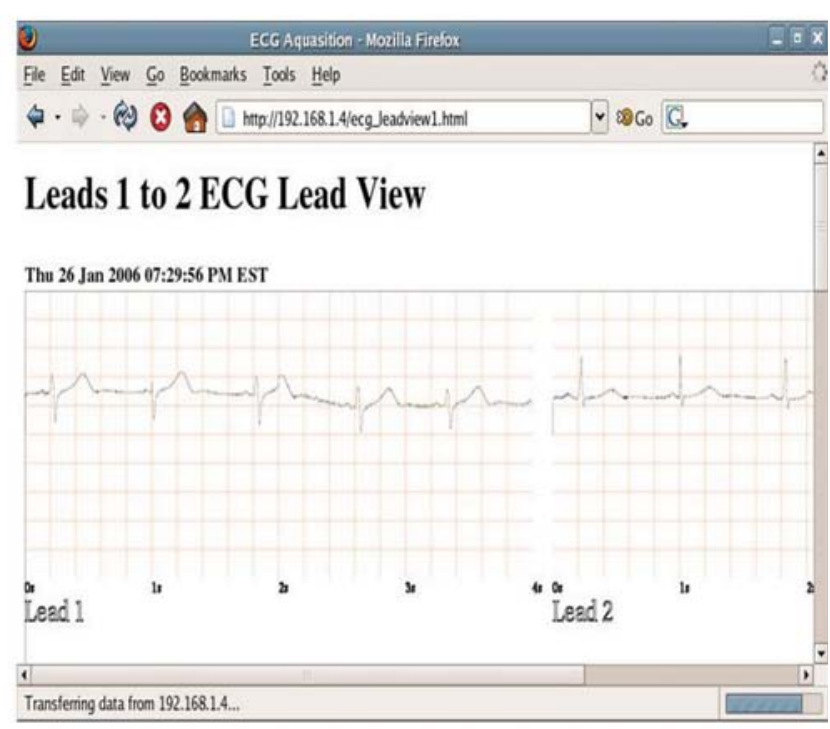

B) Current Patient ECG Instrument Output

Fig 2. MIN Webpage Interface Showing ECG leads

entered by the nurse. An example of a patient record can be seen in a web browser as seen in Fig 2 A).

Alternatively as shown in Fig. 1 each MIN can be accessed remotely via a local area network. A remote user can view and record the current output of the ECG instrument. This is useful for continuous patient monitoring over a long period of time.

\section{MEDICAL INFORMATION SYSTEM NETWORK}

An outline of the Medical Information System Network can be viewed in Fig. 1. The network is made up of Bluetooth Hotspots, distributed throughout patient wards. The Bluetooth Hotspots are implemented by Medical Information Nodes (MIN). Each MIN is connected to a patient information server by a local area network. The MIN is designed to interface to medical instruments through a serial connection. Currently the MIN is able to control and record the output of an ECG instrument. The MIN is Bluetooth enabled in order to allow control interaction with MCDs. As each MIN has a LAN connection, a webpage interface can be used to control and view the instrument output of the MIN The patient information server is used to store patient instrument readings and records which can be accessed by each MIN.

\section{A. Interactive Interface Mechanisms}

There are two interactive interface mechanisms for the medical information system: an MCD based interface and a web based interface.

\section{B. Mobile Computing Device Interactive Interface}

The MCD based interface consisted of an ECG viewer program that allows a user to interact with the medical information system using their MCD. Connection between the user's MCD and medical information node is facilitated via Bluetooth, as shown in Fig. 1 the main requirement of the MCD interface is to display real-time ECG waveforms and to control the medical information node. Two methods for transmitting and viewing the ECG waveforms, via
Bluetooth were investigated: standard image format and a custom data stream methods.

\section{Analysis of Real-time Display Methods}

There are two methods for displaying real-time waveforms either using a conventional image formats such as JPEG or a custom data format. Both methods have advantages and disadvantages. A telemedicine system described Pavlopoulos et al [8], shows how the ECG waveforms can be displayed in a conventional image format such as JPEG. The disadvantage of using image formats is the high data rate required to transfer the image files representing the ECG waveform, particularly in realtime. Sizes of image files as noted by [8] are between $2.5 \mathrm{KBytes}$ to $3 \mathrm{kBytes}$ The advantage of using standard image formats is that support for displaying images is widespread and extra information such as blood pressure or heart rate can also be conveyed in the image file.

Display application programs such as web browsers are commonly found on most mobile smart-phones. Unfortunately, the capabilities of web browsers can vary between different MCDs. In order to display a real-time image file in a web browser, JavaScript is required. It was found that JavaScript support can be limited on different mobile web browser applications due to the memory and CPU processing limitations of MCDs.

The advantage of using a custom data format is to represent an ECG waveform is that a high data rate is not required in order to transfer the ECG Lead waveform data stream. A disadvantage is that a custom program is required to view the ECG waveform. Unfortunately this means that custom viewing programs would need to be created for MCDs that differ in capabilities. This would need to be done in order to allow a variety of MCDs to be used with the medical information system.

A custom ECG viewer program was written in order to allow the real-time display of the ECG waveform. The client program was written using the Symbian Nokia S60 Software development kit [9]. The Symbian Nokia S60 


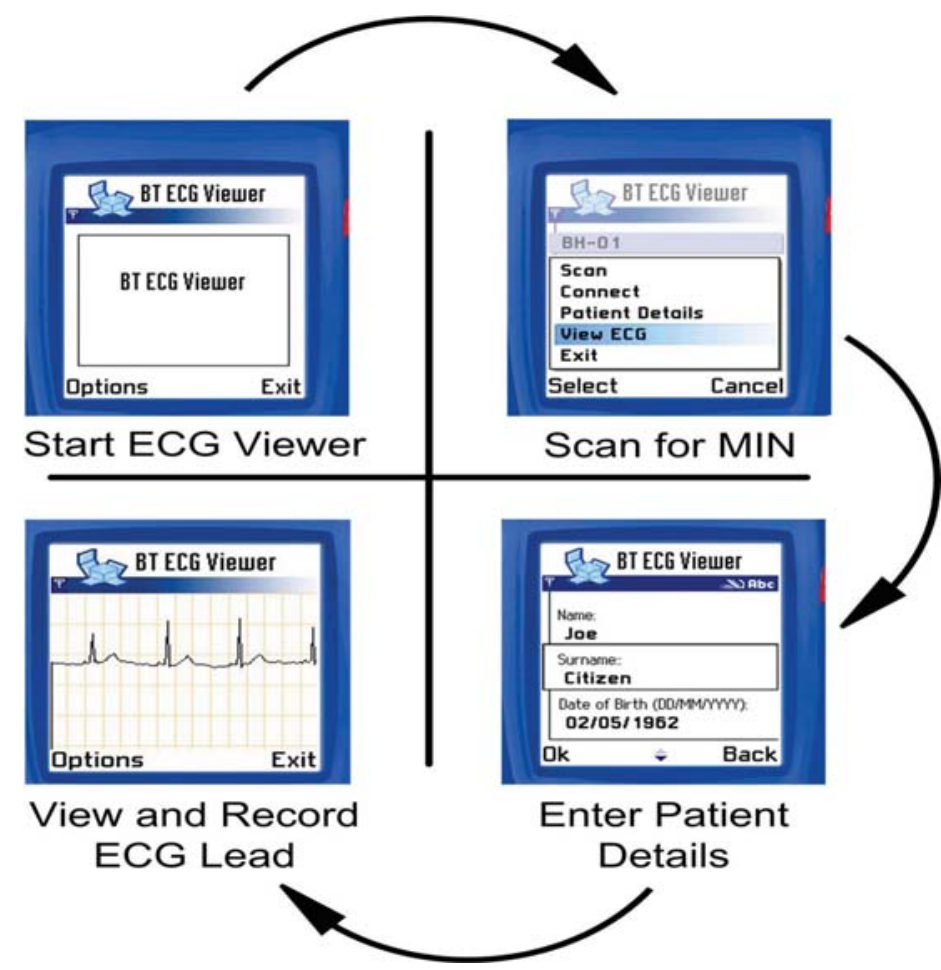

Fig. 3. Patient Form as Seen on User's Mobile Computing Device (MCD)

Bluetooth API allows the Bluetooth serial port profile to be accessed via a standard socket interface. The Symbian Nokia S60 basic drawing API allowed real-time display of the selected ECG Lead waveform.

\section{Bluetooth Connectivity}

Two Bluetooth profiles were analysed with respect to data rates. A series of tests were conducted. A comparison of average data rates for different Bluetooth profiles can be seen in Table 1. The data rate to view and ECG Lead waveform from the instrument used by the MIS is $1000 \mathrm{bps}$. Due to the higher data rate and the simplicity of setting up a connection, control communications protocol uses the Bluetooth Serial Profile as a transport layer. Setting up a dialup profile, requires a point to point protocol (PPP) connection. Although this does have the advantage of using standard TCP/IP network connections to transfer the data, it requires the MIN and MCD implement a PPP and TCP/IP connection. This can require significant memory and processing resources from both the MCD and MIN.

\section{E. Web Interface}

The Medical Information Node (MIN) is connected to a conventional LAN. The advantage of this is that a standard web browser and network connection can access, view and control the MIN, as shown in Fig 2 B). With the future addition of Wireless LAN (WLAN) connectivity, this would allow the MIN to be accessed by a variety of WLAN enabled MCDs.

The ECG instrument Patient lead waveforms can be viewed using the MIN's webpage interface. The MIN ECG lead display webpage uses a CGI script to generate a dynamic Scalable Vector Graphics (SVG) image of the received ECG lead data stream from the ECG instrument. Scalable Vector Graphics is a XML based mark-up language [10] which allows an accurate image to be drawn from a raw data stream which in this case is an ECG waveform.

The logistical advantage of having the MIN connected to a LAN is that the recorded output of the ECG instrument can be recorded in the Patient Information Server's Database. Another advantage is the use of standard web page techniques to access the instruments output. This give the MISN the capability of allowing an instrument's output to be viewed and controlled remotely other than supporting localised interaction.

\section{MEdiCAL INFORMATION NODE}

The Suzaku embedded system platform was used to implement the Medical Information Node is implemented. An outline and photo of the MIN is shown in Fig. 4 A) and B) The MIN's consists of a 32bit processor and communicates to the Bluetooth Serial Profile Module and ECG instrument via a serial interface. The Suzaku platform runs the uClinux Operating System and also has an Ethernet MAC controller to allow the MIN to be connected to a LAN. The MIN is based on a platform that was developed previously by [11]. The digital implementation of the MIN for acquiring and processing real-time ECG signals is discussed in [11].

A Bluetooth Serial Port module [12] is used to facilitate the Bluetooth link between the MIN and the mobile device.

TABLE I

Average Data Rates of Bluetooth Profiles

\begin{tabular}{|l|l|}
\hline \multicolumn{1}{|c|}{ Bluetooth Profile } & \multicolumn{1}{|c|}{$\begin{array}{c}\text { Average Data Rate } \\
(\mathrm{kbps})\end{array}$} \\
\hline Serial Port & 115 \\
\hline Dialup (PPP over Serial Port) & 15 \\
\hline
\end{tabular}




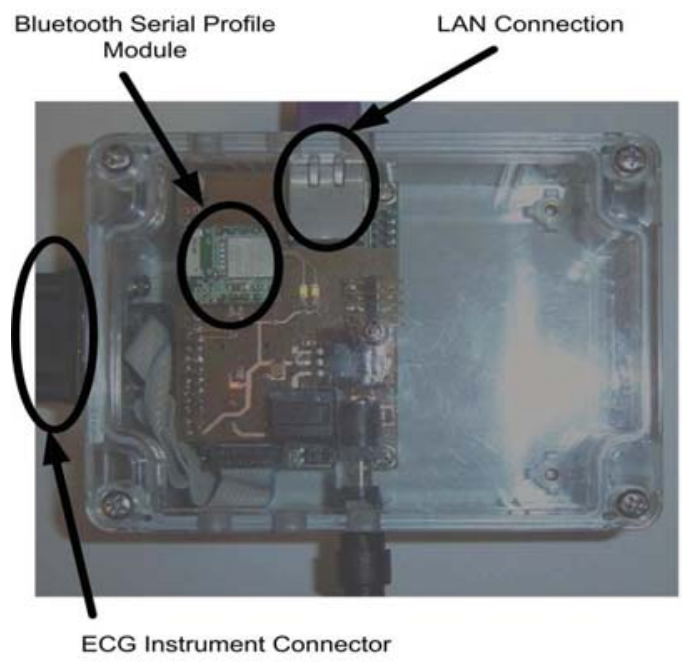

A) Medical Information Node

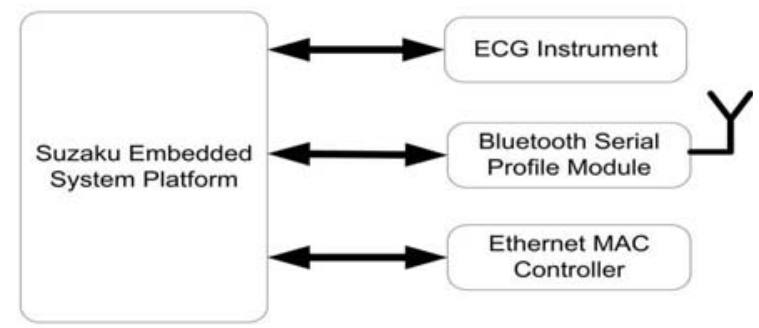

B) Outline of Medical Information Node

Fig. 4. Medical Information Node

The Bluetooth Serial Port Module is a separate device to the FPGA that functions as a Bluetooth serial port as specified by the Bluetooth Serial Port Profile Specification [4]. It is controlled by the Bluetooth controller process (described previously) through a serial UART.

An external Ethernet MAC controller is used to connect the MIN to an Ethernet network. As mentioned before, the MIN functions as a web server. When the MIN's webpage is accessed through a web browser, the Web Controller uClinux process is activated. The web controller starts the ECG instrument and displays the ECG lead data on the Webpage.

\section{MIN SOFTWARE CONFIGURATION}

Fig. 5 shows a flow diagram of the software modules that implements the functionality of the medical information node (MIN). The MIN uses the uClinux OS which is an embedded systems variant of the Linux OS. The uClinux OS is designed to operate on processors that do not have memory management units. The advantages of using uClinux are its inbuilt networking and web server hosting functions. Another advantage is that the software processes were prototyped on a Linux based PC.

Three software process entities are used to implement the functionality of the MIN. The ECG controller process is used to control the ECG instrument. The ECG controller extracts and assembles the sampled ECG lead data from the ECG instrument. Once the ECG lead data has been extracted, it is then passed to the Bluetooth and web controller processes. A socket interface is used to transfer the data and commands between the Bluetooth, web and ECG controllers.

The Bluetooth controller process is used to receive and interpret commands from the Client program on the user's MCD. If a user attempts to view an ECG lead waveform, the MCD will send a lead request command to the Bluetooth controller which will respond with a real-time stream of the requested ECG lead waveform data. The Bluetooth controller process will also save the recorded ECG data in as a medical record on the MIS using a

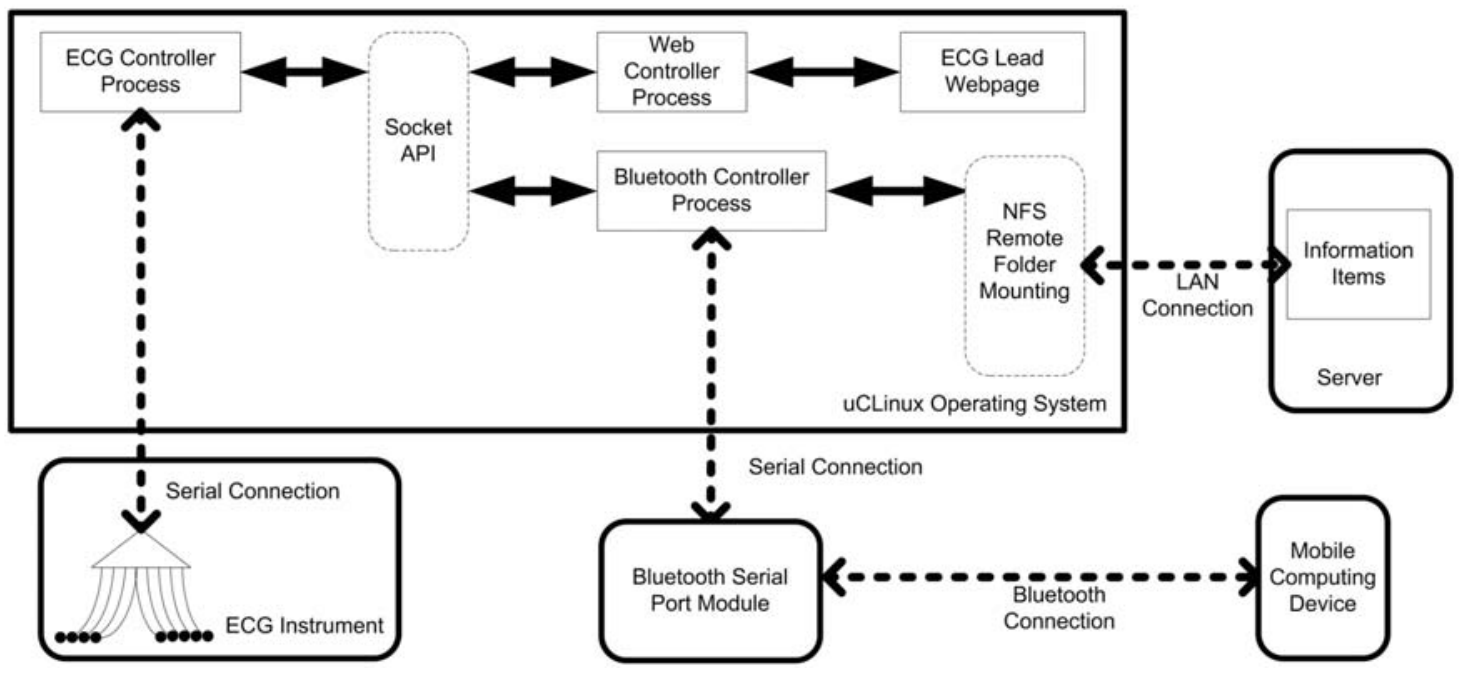

Fig. 5. Medical Information System Software Architecture 
Network File System (NFS) mounted folder via the Ethernet connection.

As described in previously, the webpage interface displays all ECG lead waveforms. The web controller process is used by the webpage interface to access the realtime ECG lead waveforms from the ECG controller process. More information about the webpage interface is described in Web Interface Section.

\section{CONCLUSIONS AND FURTHER WORK}

Our paper presented an integrated networked ECG monitoring system that uses mobile computing devices as a means of interaction. The MISN consists of medical information nodes (MINs) networked to a central server. Each MIN could be accessed by a mobile computing device via a Bluetooth connection. The MIN also allow the output of an ECG instrument to be viewed using web browser and to record the ECG instrument's output into a file that is saved on a central server. This meant that the ECG Instrument's output could be directly organized into a patient database without further human interaction.

We analyzed two methods for displaying real-time waveforms using a conventional image formats such as JPEG or a custom data format. We found that using a custom data format is to represent an ECG waveform did not require a high data rate but that a custom program was required to view the ECG waveform.

One of the advantages of using Bluetooth is that its short range ensures that the MIN only to be accessed when the user is within a particular location. This allowed the MIN to provide location specific access to medical instruments depending on where the user is. We also analyzed the Bluetooth Serial Port and Dialup profiles for streaming the real-time ECG waveform data. We found that Bluetooth Serial Profile was ideal to use because it allowed a higher data rate and required less complexity of setting up a connection between the MIN and the MCD.

The MIN was a uClinux OS based platform which allowed it to interface easily to an existing LAN. Although the MISN was successfully tested, further work is required to increase the capability of the MISN by expanding the MINs to interface to other medical instruments. Wireless
LAN connectivity will also be investigated for use with MIN platform.

\section{REFERENCES}

[1] T. E. Bell, "Medical Records: From Clipboard to Point-and-Click," IEEE The Institute, vol. 29, 2005.

[2] "Standard Communications Protocol for ComputerAssisted Electrocardiography," European Committee For Standardization, 2000.

[3] P. Schluter, T. Cooper, K. Hassing, D. Nowicki, L. Steubesand, and W. Silver. http://www.ieee1073.org/standards/1107330300/11073-30300.html: IEEE 1073, 2005.

[4] Bluetooth SIG, "Bluetooth Specification Documents."

[5] M. F. A. Rasid and B. Woodward, "Bluetooth telemedicine Processor for multichannel biomedical signal transmission via mobile cellular networks," Information Technology in Biomedicine, IEEE Transactions on, vol. 9, pp. 35-43, 2005.

[6] K. Hung and Y.-T. Zhang, "Implementation of a WAP-based telemedicine system for patient monitoring," Information Technology in Biomedicine, IEEE Transactions on, vol. 7, pp. 101-107, 2003.

[7] R. Aston, Principles of biomedical instrumentation and measurement. Columbus: Merrill Pub. Co., 1990.

[8] S. Pavlopoulos, E. Kyriacou, A. Berler, S. Dembeyiotis, and D. Koutsouris, "A novel emergency telemedicine system based on wireless communication technology-AMBULANCE," Information Technology in Biomedicine, IEEE Transactions on, vol. 2, pp. 261-267, 1998.

[9] "Series 60 Developer Platform." http://www.forum.nokia.com/main/resources/docume ntation/s60.html: Nokia Corporation, 2007.

[10] A. Quint, "Scalable vector graphics," Multimedia, IEEE, vol. 10, pp. 99-102, 2003.

[11] M. D'Souza, M. Ros, and A. Postula, "Wireless Medical Information System Network for Patient ECG Monitoring," presented at Digital System Design: Architectures, Methods and Tools, 2006. DSD 2006. 9th EUROMICRO Conference on, 2006.

[12] "Promi-ESD-02 User Manual Version 1.1." www.initium.co.kr: Initium, 2004. 